\title{
Marden Walker Syndrome
}

\author{
Matijević Valentina $^{{ }^{*}}$, Goranka Radmilović ${ }^{2}$, Kraljević Marija ${ }^{1}$, Barbarić Bernarda ${ }^{1}$, Petra \\ Matijević $^{3}$, Željka Kolak ${ }^{4}$ \\ ${ }^{1}$ Clinical Department of Rheumatology, Physical Medicine and Rehabilitation, Sestre milosrdnice University \\ Hospital Centre in Zagreb; Josip Juraj Strossmayer University of Osijek - Faculty of Medicine in Osijek; \\ Croatia \\ ${ }^{2}$ Daruvarske toplice, Daruvar; Josip Juraj Strossmayer University of Osijek - Faculty of Medicine in Osijek; \\ Croatia \\ ${ }^{3}$ student Dental Medicine, University of Zagreb; Croatia \\ ${ }^{4}$ The General County Hospital Vinkovci, Vinkovci, Croatia
}

*Corresponding Author: Valentina Matijević, Clinical Department of Rheumatology, Physical Medicine and Rehabilitation, Sestre milosrdnice University Hospital Centre in Zagreb; Josip Juraj Strossmayer University of Osijek-Faculty of Medicine in Osijek; Croatia, Email: valentina.matijevic@ gmail.com

\begin{abstract}
Marden Walker syndrome is a syndrome whose underlying pathological mechanism has not been clearly established yet. It is assumed that it is a developmental disorder of the central nervous system which is inherited in an autosomal recessive manner. Three main characteristics and diagnostic criteria of the syndrome are blepharophymosis, congenital joint contractures and mask-like face. Numerous of other anomalies have been described and the exact gene that causes the disease is unknown. We present a thirteenyear-old girl with MWS and pain on the right side of the face to the ear. Medical repeatedly examination was not possible because of the strong spasm of orofacial musculature, as well as the examination of the oral cavity and the teeth. Vojta principles were applied during five days in the treatment, resulting with reduced spasm in orofacial musculature thus enabling the dentist to examine the child. This case report highlights the importance of continuous psychomotor stimulation because of prevention, progression and complications of deficits, as the importance of evaluating motor development and creating specific rehabilitation plan for each child. In this way we try maximizing the quality of child's life in accordance with the possibilities in everyday life of the parents and the child.
\end{abstract}

Keywords: Marden Walker Syndrome; Developmental disorder; psychomotor stimulation;

Abbreviations: Marden Walker Syndrome (MWS)

\section{INTRODUCTION}

Marden-Walker syndrome is a developmental disorder of the central nervous system, characterized by multiple contractures as decreased muscular mass, kyphoscoliosis and typical facial features (mask face expression with blepharophimosis, micrognathia, cleft or high-arched palate, low-set ears) [1]. It is believed that this disorder is inherited in an autosomal recessive manner and the most obvious signs are present in the neonatal period [2].

One case report of MWS in India writes about unusual manifestation of neurological, cardiovascular, and genitourinary system [3]. Hydrocephalus, hypolastic corpus callosum, cerebellar vermis hypoplasia and enlarged cisternal magna are associated with microcephaly and is very common cerebral malformations in a patient with MWS [4].

The MWS was reported initially in 1966 by Marden and Walker [5]. They described a female infant with multiple contractures (micrognathia, immobile facies, kyphoscoliosis, limb contractures, pigeon breast, and arachnodactyly).

Here is described a case study of a patient with MWS after carrying out an intense Vojta therapy with a physiotherapist.

There are not so many researches about importance of multidisciplinary co-operation among various experts when it comes to patients with MWS [6]. The team of experts may include a paediatrician, a neurologist, specialist in 
physical medicine and rehabilitation, physiotherapist, dentist, speech therapist and psychologist, in order to stimulate further psychomotor development [7].

The goals of multimodal rehabilitation of the child with MWS are the most encouragement of all ability in accordance with psychophysical characteristics and life expectancy [8]. To enable intellectual, physical, emotional and social development and to ensure the conditions for successful integration into daily life with the greatest possible potential of independence and successful socialization.

\section{Case Study}

We reported on a girl (13) with MWS who after birth had vesicoureteral reflux, splenomegaly, epilepsy, severe psychomotor retardation, dysmorphic head, micrognathia and weakness of musculature. Our patient has been included into the psychomotor stimulation since third month of life. At the age of two, patient could not swallow, talk, sit, and walk. She had dysfunction fists and was incontinent.

Psychomotor stimulation aims to prevent the progression of incurred deficits and occurrence of complications in order to maximize the quality of life and independence, in accordance with the possibilities of participation into the social community [9]. Early psychomotor stimulation included medical gymnastics with conventional program and Bobath Concept, parental counselling by speech therapists and psychologists [7]. The patient attended a onetime gymnastic exercise on a weekly base and intensively practiced with her mother at home according to the instructions of a physiotherapist. Every 2 to 3 months the patient was subjected to a detailed assessment to a specialist in physical medicine and rehabilitation. Also, it was additionally involved in speech therapy as part of kindergarten stay.

The child's doctor specialist in physical medicine and rehabilitation evaluates the spontaneous motor skills, active motions, qualitative analysis of general movement, and the strength of the muscles, palpation and passive motility testing, reflexes testing by the Vojta principle [7]. Doctor specialist in physical medicine and rehabilitation should have work experience in this area for at least five years or certification for completing the course by Vojta principles [7].

The physiotherapist encourages the proper pattern about the movement of the child, but at the same time raises the child's neuro physiological development which is needed for the child to carry out the wanted or desired movement [7].

Speech and language therapist estimates the speech-language and communication development. Based on standardized tests, the speech and language therapist evaluates understanding and speech in children with motor distortions. Speech therapist examines the orofacial muscle weakness associated with difficulties with sucking, swallowing and chewing, as it is the case for children with MWS. As a result of orofacial weakness, there are also difficulties in articulation and fluency in speech [10].

The psychologist evaluates the child's development of psycho motoric skills. According to the development gives advices to the parents on educational procedures and behaviour about the child's deviations in motor and neurophysiological development. In addition, psychologist uses various types of tests to measure the general intelligence and readiness for school [7].

Medical examination established palpatory pain in the area of the temporomandibular joint (right). The function of the temporomandibular joint could not be investigated because of the strong spasm of orofacial musculature, as well as the examination of the oral cavity and the teeth.

Therefore, it was indicated the conduct of Vojta therapy as to reduce the tone of the muscle and examine the function of the temporomandibular joint and possibility of examination by the dentist. This therapy was applied intensively during five days.

\section{RESULTS AND DISCUSSION}

After five days of Vojta therapy, child's doctor specialist in physical medicine and rehabilitation found a neat function in temporomandibular joint. Our patient was not only enable to open her mouth, but also neglected the intense pain she felt over a few days. Also, this girl chewed and swallowed food more easily and spoke more fluently. After carrying out intensive Vojta therapy, the dentist made a detailed review and diagnosed inflammation of the gingiva in the upper jaw area (right).

This case report, speaks in support of the importance of carrying out Vojta therapy in patients with MWS.

The Vojta concept is based on early kinesiological diagnosis based on spontaneous motion and alignment, posture, and primitive 
reflex estimation [11]. Based on the estimation, an individual plan and program of the therapy was based on beginning of reflex, stimulation of zones and pressures in certain positions are developed to cause coordinated complexes that are otherwise created and stored in the central nervous system in a child [11]. A very important factor of the Vojta concept is in the education of family members supervised by physiotherapists, to enable the child to regularly perform therapy several times per day in order to obtain the best possible results [11].

\section{Conclusion}

The objective of this case study is to highlight the need of proper counselling and treatment of patients with MWS to prevent such recurrences in the future. From our experience of Vojta therapy, positively affects the reduction of spasm in the area of orofacial muscularity in children with MWS, as soon as the temporomandibular joint and other joints on the body are easier to mobilize.

These findings further delineate the syndrome and suggest that there is always an important multimodal approach and cooperation between specialists in different professions to improve the quality of patient's life with MWS in their daily lives and prevent complications. The desire is to find a common solution and to work on linking services in multimodal rehabilitation of patient with Marden Walker syndrome.

\section{REFERENCES}

[1] D. Heron, "Marden-Walker syndrome," Orphanet Encyklopedia, November 2003.

[2] Marden P.M., Walker W.A., A new generalized connective tissue syndrome, Am J Dis Child, ch. 112, 225-8(1966)
[3] Taksande A.M., Vilhekar K.Y., Unusual manifestation of Marden-Walker syndrome, Indian J Hum Genet, 18(2), 256-258(2012).

[4] García-Alix A., Blanco D., Cabañas F., Garcia Sanchez P., Pellicer A., Quero J., Early neurological manifestation and brain anomalies in Marden-Walker syndrome, Am J Med Genet, 44(1), 41-5(1944).

[5] Gossage D., Perrin J. M., Brief Clinical Report and Review: A 26-Month-Old Child With Marden-Walker Syndrome and Pyloric Stenosis, Am J Med Genet, 26(4), 915919(1987).

[6] Charles R. K., Magenis E., The Marden-Walker Syndrome, J Med Gen, 15, 366-369(1978).

[7] Matijević V., Marunica Karšaj J., (Re)habilitation guidelines for children with neurodevelopmental disorders, Fiz. rehabil. med, 27 (3-4), 302-329(2015).

[8] Matijević V., Košiček T., Crnković M., Radanović B., The participation of children with neurodevelopmental risk factors in the early rehabilitation program in relation to the level of parental education, Acta Clin Croat, 50, 457-61(2011).

[9] Matijević V., Šečić A., Šafran I., Estimation of the difference in the effectiveness of neurodevelopmental stimulation programs in stationary and ambulatory conditions, Fiz. rehabil. med., 28 (1-2), 181-192(2016).

[10] Matijević V., Kraljević M., Therapeutic approach: orofacial-motor exercises in the rehabilitation of muscular dystrophy. In: Filodiritto, editor. 1st ed. Italy: Bologna; 1722(2017).

[11] Jung M.W., Landenberger M., Jung T., Lindenthal T., Philippi H., Vojta therapy and neurodevelopmental treatment in children with infantile postural asymmetry: a randomised controlled trial, J Phys Ther Sci, 29(2), 301306(2017).

Citation: Matijević Valentina, Goranka Radmilović, Kraljević Marija, Barbarić Bernarda, Petra Matijević, Željka Kolak. et al. Marden Walker Syndrome. ARC Journal of Gynecology and Obstetrics 2017; 2(3):1-3. DOI: dx.doi.org/10.20431/2455-9792.0203001

Copyright: (C) 2017 Authors. This is an open-access article distributed under the terms of the Creative Commons Attribution License, which permits unrestricted use, distribution, and reproduction in any medium, provided the original author and source are credited. 